\title{
Can Residual Symptoms During Inter-Episode Period after Partial Remission in Bipolar I Disorder Have Cyclic Patterns with Specific Frequencies?
}

\author{
YongJun Cheon', Eunsoo Moon ${ }^{2,3} \bowtie$, Je-Min Park ${ }^{2,3}$, Byung-Dae Lee ${ }^{2,3}$, Young-Min Lee ${ }^{2,3}$, \\ Hee-Jeong Jeong ${ }^{2}$, Tae-Uk Kang ${ }^{2}$, Jeonghyun Park' ${ }^{2}$, and Yoonmi Choi ${ }^{2}$ \\ ${ }^{1}$ Department of Psychiatry, Dongrae Hospital, Busan, Republic of Korea \\ 2Department of Psychiatry, Pusan National University Hospital, Busan, Republic of Korea \\ ${ }^{3}$ Department of Psychiatry, Pusan National University School of Medicine, Busan, Republic of Korea
}

This case report aimed to describe cyclic patterns of residual mood symptoms in partially remitted bipolar I patient. In a 24-year-old woman with bipolar I disorder, residual mood symptoms measured by self-rated daily mood chart for 18 months were analyzed using wavelet analysis. A 146-day periodicity was prominent for the first 100 days after discharge. Between 100-200 days, 146-day periodicity was progressively diminished and 21- and 8-day periodicity was prominent. Between 200-516 days, 21-day periodicity was diminished and 85-day periodicity became prominent. This case suggest that bipolar patients might have cyclic residual symptoms with specific frequencies.

Psychiatry Investig 2018;15(3):330-334

Key Words Bipolar disorder, Rapid cycling, Cyclicity, Residual symptom, Wavelet analysis.

\section{INTRODUCTION}

Bipolar disorder is a recurrent and chronic disease that has wax and wane in the long-term course. ${ }^{1}$ Longitudinal course of bipolar disorder is known to have various patterns, in some cases, consisting of completely subsided and partially remitted having residual symptoms during inter-episode. ${ }^{2}$ Residual symptoms can be highly associated with relapse and recurrences of mood episode. ${ }^{3}$ These residual symptoms impose a negative impact on cognitive functions and functional status, and clinical importance is increasing., ${ }^{2,4}$ Patterns of residual symptoms have sometimes mild depressive symptoms with relatively long period, and have alternative course between mild depressive and hypomanic symptoms, and enhanced positive and negative emotional reaction according to life

Received: May 26, 2017 Revised: August 18, 2017

Accepted: August 23, 2017

$\triangle$ Correspondence: Eunsoo Moon, MD, PhD

Department of Psychiatry, Pusan National University Hospital, Pusan National University School of Medicine, 179 Gudeok-ro, Seo-gu, Busan 49241, Republic of Korea

Tel: +82-51-240-7303, Fax: +82-51-248-3648, E-mail: esmun@hanmail.net

(a) This is an Open Access article distributed under the terms of the Creative Commons Attribution Non-Commercial License (http://creativecommons.org/licenses/bync/4.0) which permits unrestricted non-commercial use, distribution, and reproduction in any medium, provided the original work is properly cited. events. ${ }^{4,5}$ However, there is no study about the cyclicity of residual mood fluctuation yet. Most studies analyzed the patterns and instability of mood changes for several months. Only one study examined mood states for 2 years, however, that study measured monthly mood state, and did not analyze the cyclicity of residual mood symptoms. Therefore, we postulated that inter-episode mood changes in some bipolar patients might have some specific cyclicity, and the changes over the time. Fortunately, we observed the cyclic patterns of mood fluctuation for 18 months in partially remitted bipolar I patients. We report our single case report about analyzing the cyclicity of residual mood changes using wavelet analysis.

\section{CASE}

Ms. A is a 24-year old college student with a psychiatric history of DSM-5 defined bipolar I disorder. She was admitted for 2 months in psychiatric ward with her first manic episode on euphoric mood, mood fluctuation, hyperactivity, talkativeness, decrease need for sleep, grandiose delusion, and auditory hallucination when she was 16 years old. At first admission, she was taking lithium $900 \mathrm{mg}$, valproate $750 \mathrm{mg}$, olanzapine $5 \mathrm{mg}$. After discharge, she undergo three additional 
admissions due to recurrences of three manic episodes with psychotic features at age 19,21, and 22, because she did not taking medication regularly. At 4th admission, she showed treatment-resistant severe manic symptoms with mood fluctuations. After admission for 85 days, she was partially remitted on taking combination of medications showing partial response, such as lithium $900 \mathrm{mg}$ (blood level: $0.67-0.82$ $\mathrm{mmol} / \mathrm{L}$ ), valporate $1,000 \mathrm{mg}$ (blood level: $93.01-106.90 \mathrm{ug} /$ $\mathrm{mL}$ ), risperidone $5 \mathrm{mg}$, quetiapine $100 \mathrm{mg}$, propranolol $40 \mathrm{mg}$, benztropine $1 \mathrm{mg}$.

After discharge, she exhibited residual mood fluctuation and the changes of mood-related behaviors such as social withdrawal, excessive dancing, excessive exercise over followup in the outpatient clinic. After temporary exacerbation, she became recovered, through mood fluctuation, gradually, she was improved. However, she did complaints of adverse effects of medications such as alopecia, rigidity, drooling, gait disturbance, dizziness, binge eating, daytime somnolence. Her medications were gradually reduced and changed into lithium 300 $\mathrm{mg}$, valproate $500 \mathrm{mg}$, risperidone $3.5 \mathrm{mg}$, ziprasidone $20 \mathrm{mg}$, propranolol $40 \mathrm{mg}$ bid, benztropine $1 \mathrm{mg}$. She had obsessive compulsive personality, and recorded daily mood rating, sleep duration, weight and menstrual period using mood and sleep chart. Her mood fluctuation was various and complex. It was difficult to analyze the pattern of mood changes with the naked eyes. Therefore, we analyzed the pattern of changes as well as the existence of rhythmicity in mood and sleep records us- ing wavelet transform. Various extrinsic and intrinsic factors can influence biological process, thus biological signals such as mood and sleep records are non-stationary. And wavelet transform is known to be powerful for obtaining amplitudes at multiple time scales from these data. Continuous wavelet transform was performed on her self-reported mood chart and sleep time using Morlet wavelet (Figure 1) as mother wavelet on Matlab R2013b.

Meanwhile, changes in dominant frequencies had some relevance with the change of clinical symptoms. For example, at Period A (between 0-100 days), wavelet analysis showed dominance of 146-day periodicity and patient showed great fluctuations of residual symptoms right after discharge. At period B (between 100-200 days), 146-day periodicity was progressively diminished and 21- and 8-day periodicity was prominent. At that time, patient showed mixed symptoms (CGI-BP-M $\geq 3$ at 126, 130th day and CGI-BP-D $\geq 4$ at 134, 148 , 195, and 202th day). Finally, at period C (between 200516 days), when patient gradually recovered and became stable, 21 -day periodicity was diminished and 85 -day periodicity became prominent.

\section{DISCUSSION}

This case showed that a bipolar patient has cyclic components of daily mood chart recording during inter-episode period. These findings suggest that residual mood symptoms of

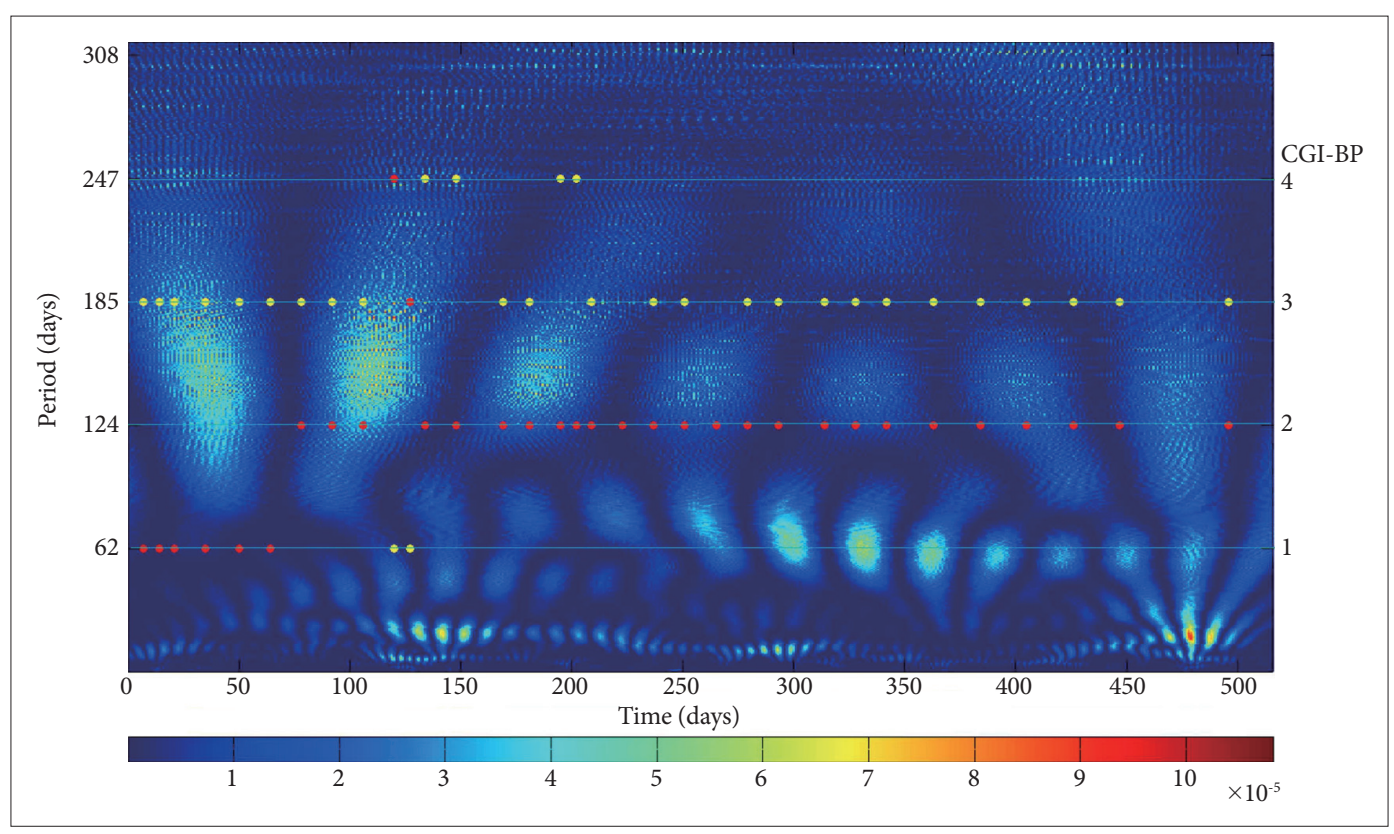

Figure 1. Continuous wavelet transform scalogram on patient's self mood rating. Horizontal axis represents time in days. Vertical axis represents scale factors of the wavelet, which is converted to period of mood cycle. Color of the scalogram represents intensity of the corresponding wavelet coefficient (blue: low, red: high). As noted, four kinds of high intensity bands can be identified (cycles with period of 146, 85, 21, and 8 days). Red dot: CGI-BP-M (Score 1-4), Yellow dot: CGI-BP-D scores (score 1-4). CGI-BP-M: Clinical Global Impression Bipolar Version-Mania, CGI-BP-D: Clinical Global Impression Bipolar Version-Depression. 
longitudinal course in patients with bipolar disorder might have cyclic patterns with specific frequency. Cyclic patterns might be composed of several components with specific fre- quency, such as several months (around 146 days and 85 days), about 1 months (around 21 days), about 1 weeks (around 8 days). We adopted wavelet analysis methodology to analyze
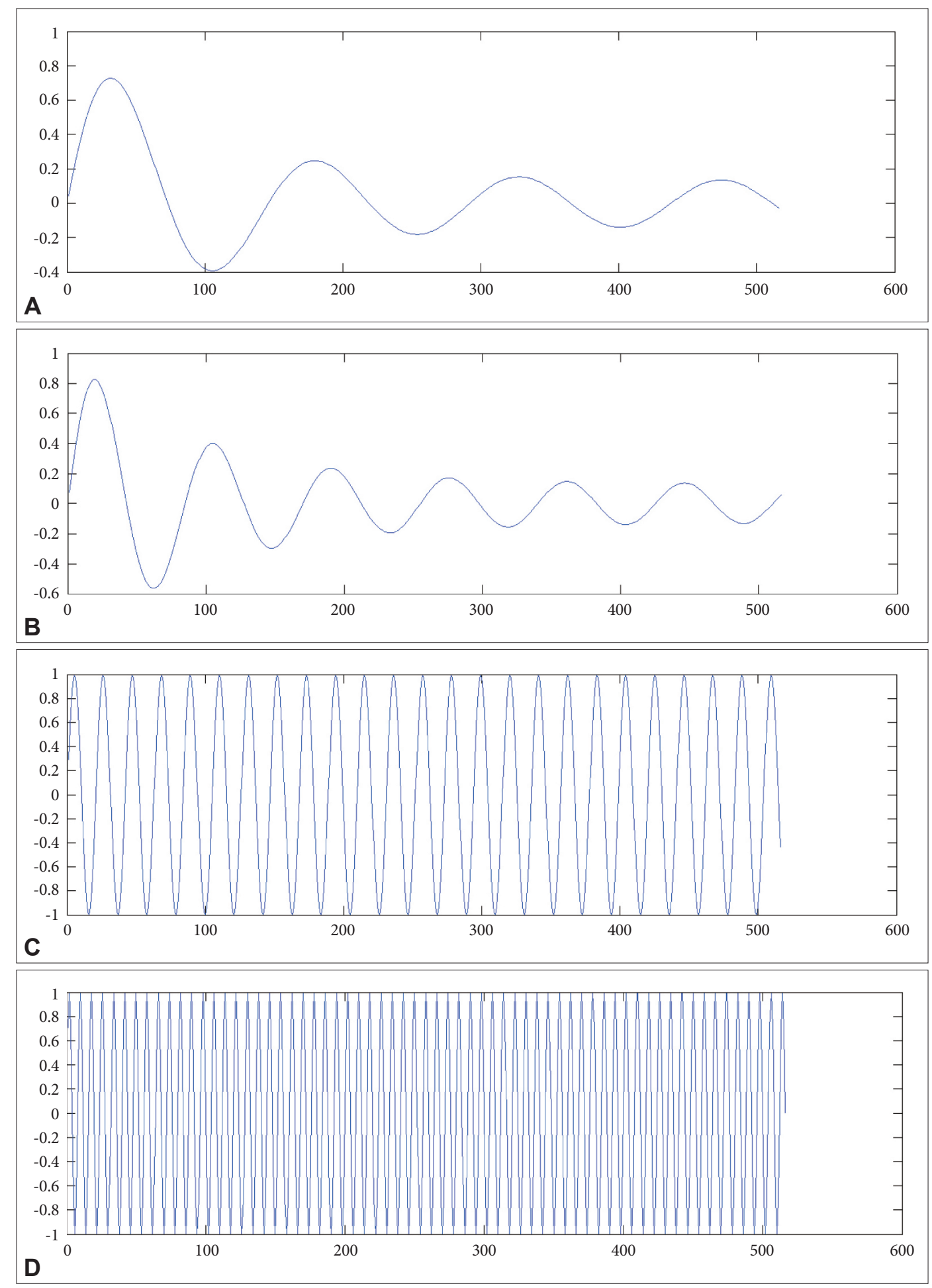

Figure 2. (A) Dampened sinusoidal wave with period of 146 days. (B) Dampened sinusoidal wave with period of 85 days. (C) Sinusoidal wave with period of 21 days. (D) Sinusoidal wave with period of 8 days. (E) Sum of A-D. (F) Wavelet scalogram on E. Wavelet scalogram of sum of four sinusoidal waves with different period shows four different intensity bands, and signal intensity (=color) on the scalogram correlates to amplitude of corresponding waves. Similarities between Figures 1 and $2 \mathrm{~F}$ visualizes implication of wavelet scalogram of patient's mood rating scales. 

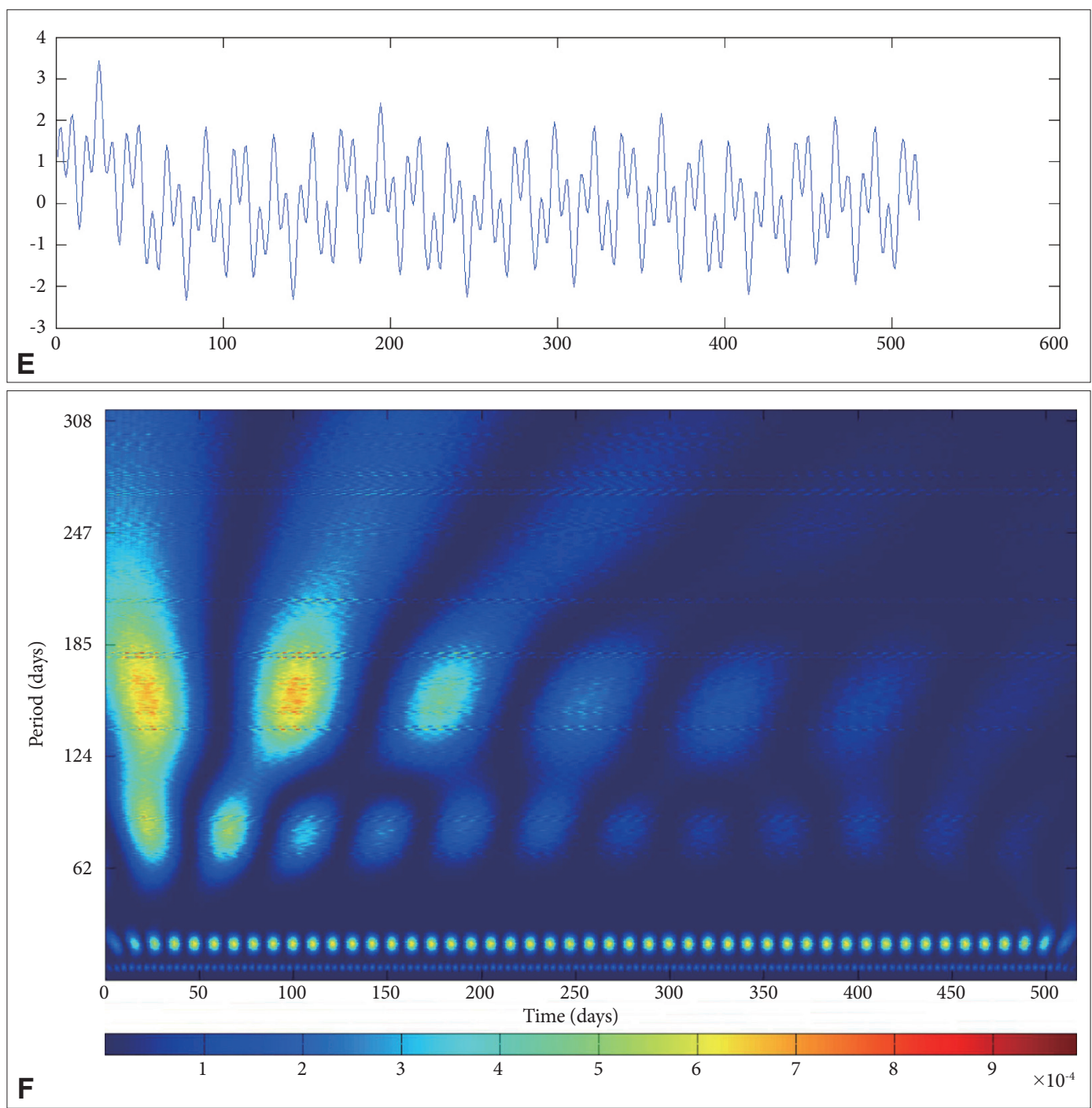

Figure 2. (A) Dampened sinusoidal wave with period of 146 days. (B) Dampened sinusoidal wave with period of 85 days. (C) Sinusoidal wave with period of 21 days. (D) Sinusoidal wave with period of 8 days. (E) Sum of A-D. (F) Wavelet scalogram on E. Wavelet scalogram of sum of four sinusoidal waves with different period shows four different intensity bands, and signal intensity (=color) on the scalogram correlates to amplitude of corresponding waves. Similarities between Figures 1 and $2 \mathrm{~F}$ visualizes implication of wavelet scalogram of patient's mood rating scales.

periodicity of bipolar patient's subject mood score. To demonstrate its implication more clearly, we modeled four kinds of sine waves with different frequencies $\left(146^{-}, 85^{-}, 21^{-}\right.$, and 8 days) and conducted wavelet analysis on combination of these sine waves (Figure 2). Consequent scalogram showed similar periodicity of patient's mood analysis, except there was modest wax and wane pattern on periodicity of patient's scalogram. It could be the reflection of the wax and wane pattern of patient's residual symptom.

And we could observe CGI-BP score was estimated as 4 for five times around 100-230 days. This could be interpreted as an emergence of new mood episode. But according to patient's history, period of elevated or depressed mood which was enough to be CGI-BP score 4 was no more than 1-2 days, which could not meet diagnostic criteria of mood episode.

In this single case report, we do not conclude the clinical meaning of these specifc frequencies. However, we can speculate that frequency around several months seems to be rapid cycling and/or seasonal patterns. ${ }^{6}$ Also, frequency around 1 month (21 days in our case) might be the reflection of menstrual cycle observed in the female patient. ${ }^{7.8}$ In addition, frequency around 8 days may be the cyclothymic component. ${ }^{9,10}$ And, these frequencies might be influenced by mood state, and gradually subsided over time by the long-term effect of mood stabilizers, though, in the acute stage, they did not show the full efficacy.

In this case report, we could observe the correlation between clinical presentation and periodicities in scalogram. 
Generally, as the severity and variability of residual symptoms diminished, dominant frequency of scalogram increased. Interestingly, when patient was at mixed state, low frequency component (145-day) became less dominant and high frequency component (21- and 8-day) became more dominant. There may be reciprocal inhibition between low-frequency and high-frequency components.

Bipolar disorder is known to show circadian rhythm abnormality. There are several kinds of biological oscillators in the brain. And they are interconnected and regulate each other, so whole brain system exhibits limited, regulated biological rhythms. But, in the state of disease, these interconnections might be out of control. Each oscillator affects mood more directly, so stability of whole brain system decreases and mood variability may be greatly increased. This case suggests that regulated biological rhythms in healthy state might be dyscontrolled and expressed in disease state. Extreme type of these dyscontrolled biological rhythms might be mood episode. In clinical situation, dyscontrolled rhythms can be different according to the type of vulnerability, therefore, there might be the various patterns of residual symptoms. These cyclic mood changes can show the self-limited course according to own frequency of mood rhythm. Mood stabilizer can reduce the amplitude of these cyclic mood swings, and improve the ability of controlling the several mood components. And these states may be reflected in changes of number of periodicities in mood scalogram.

This case report has some limitations. These findings in single case report have some problems to be generalized to bipolar patients. In addition, this case report is based on a subjective self-report of mood state in a bipolar patient, not on objective biological rhythms. Further analysis of cyclic patterns using wavelet analysis is needed to be applicable to other biological rhythm using a larger sample of bipolar patient and objective biological data obtained from wearable devices. How- ever, this case suggests that some bipolar patients might have cyclic residual symptoms, and have several cyclic components with specific frequencies. Therefore, analysis of the cyclic components of residual mood symptoms can be needed to study the longitudinal course of bipolar disorder. Furthermore, the changes in the periodicity of cyclic components for long-term treatment might be used as a state marker reflecting the longitudinal treatment response of bipolar disorder in clinical settings.

\section{REFERENCES}

1. Judd LL, Akiskal HS, Schettler PJ, Endicott J, Maser J, Solomon DA, et al. The long-term natural history of the weekly symptomatic status of bipolar I disorder. Arch Gen Psychiatry 2002;59:530-537.

2. Judd LL, Akiskal HS, Schettler PJ, Endicott J, Leon AC, Solomon DA, et al. Psychosocial disability in the course of bipolar I and II disorders: a prospective, comparative, longitudinal study. Arch Gen Psychiatry 2005;62:1322-1330.

3. Judd LL, Schettler PJ, Akiskal HS, Coryell W, Leon AC, Maser JD, et al. Residual symptom recovery from major affective episodes in bipolar disorders and rapid episode relapse/recurrence. Arch Gen Psychiatry 2008;65:386-394.

4. Gershon A, Eidelman P. Inter-episode affective intensity and instability: predictors of depression and functional impairment in bipolar disorder. J Behav Ther Exp Psychiatry 2015;46:14-18.

5. Koenders MA, Giltay EJ, Hoencamp E, Elzinga BM, Spinhoven P, Spijker AT. The bidirectional impact of perceived and enacted support on mood in bipolar outpatients: a two-year prospective study. Compr Psychiatry 2015;60:59-67.

6. Geoffroy PA, Bellivier F, Scott J, Etain B. Seasonality and bipolar disorder: a systematic review, from admission rates to seasonality of symptoms. J Affect Disord 2014;168:210-223.

7. Endicott J. The menstrual cycle and mood disorders. J Affect Disord 1993;29:193-200.

8. Parry BL, Newton RP. Chronobiological basis of female-specific mood disorders. Neuropsychopharmacology 2001;25(5 Suppl):S102-S108.

9. Akiskal HS, Hantouche EG, Allilaire JF. Bipolar II with and without cyclothymic temperament: "dark" and "sunny" expressions of soft bipolarity. J Affect Disord 2003;73:49-57.

10. Van Meter AR, Youngstrom EA, Findling RL. Cyclothymic disorder: a critical review. Clin Psychol Rev 2012;32:229-243. 